\title{
Study of suprathermal electron dynamics during ECCD using hard X-ray measurements in the TCV tokamak
}

\author{
D. Choi ${ }^{1}$, S. Coda ${ }^{1}$, J. Decker ${ }^{1}$, J. A. Cazabonne ${ }^{1}$, \\ Y. Peysson ${ }^{2}$, and the TCV team*1 \\ ${ }^{1}$ Ecole Polytechnique Fédérale de Lausanne (EPFL), Swiss Plasma Center (SPC), \\ CH-1015 Lausanne, Switzerland \\ ${ }^{2}$ CEA, IRFM, F-13108, Saint-Paul-lez-Durance, France
}

\begin{abstract}
Suprathermal electron dynamics in real and momentum spaces plays an important role in the physics of Electron Cyclotron Current Drive (ECCD), in particular for predicting the wave driven current. In the TCV tokamak, a hard X-ray spectroscopy system equipped with digital pulse detection enables detailed studies of the creation and relaxation of suprathermal electrons. The time evolution of hard X-ray spatial profiles and energy spectra is observed utilizing a minimally perturbative ECCD modulation technique. Time-dependent Fokker-Planck modeling combined with a hard X-ray synthetic diagnostic is used to interpret experimental results and test various suprathermal electron transport models. The comparison suggests a dependency of the radial transport on the EC wave power.
\end{abstract}

\section{Introduction}

Electron Cyclotron Current Drive (ECCD) induces plasma current by the resonant interaction of EC waves and suprathermal electrons, creating a toroidally asymmetric electron distribution function. With an ability to drive spatially localized currents [1,2], ECCD is used to control plasma instabilities such as the neoclassical tearing mode (NTM) $[3,4]$ and the sawtooth instability [5]. In ITER, ECCD is selected as the main stabilization tool for disruptive NTMs $[6,7]$.

To estimate the plasma current driven by EC waves, Fokker-Planck calculations of the electron distribution function have been combined with ray tracing of wave propagation. Facing a systematic over-estimation of the ECCD current, the radial transport operator in the FokkerPlanck equation has been controlled in the simulations, in order to match experimental observations [8-12], assuming the possibility of significant anomalous transport of suprathermal electrons. The effect of radial transport was supported by hard X-ray profile measurements, showing that the bremsstrahlung radiation of suprathermal electrons featured a broader profile than the EC wave absorption profile [13].

Recently the effect of EC wave scattering has been addressed as another possible mechanism that degrades the current drive efficiency and localization [14-19]. Combining wave scattering and Fokker-Planck calculations indicates that density fluctuations near the edge of the plasma

\footnotetext{
*See author list of S. Coda et al 2019 Nucl. Fusion 59112023
} 
can deflect or scatter the beam, and broaden the driven current profile in accordance with experimental observations [20,21]. As of today, it is still not clear whether anomalous transport or wave scattering is the dominant cause for the broadening of current profile and the reduction in ECCD efficiency that are observed experimentally. This question is partly due to the difficulty in observing suprathermal electron dynamics in the real and velocity spaces simultaneously.

The hard X-ray diagnostic has been a main method to analyze the energy spectrum and spatial distribution of suprathermal electrons generated by RF waves [13,22-24]. However, due to the continuum nature of the bremsstrahlung radiation [25] and the line-integrated nature of the measurement, it is not possible to obtain the electron distribution function directly from the hard X-ray measurement. This limitation can be overcome by resorting to modeling: in particular, a hard X-ray synthetic diagnostic coupled to Fokker-Planck modeling allows a direct comparison between measurement and simulation [21,24,26, 27].

To investigate suprathermal electron dynamics in both configuration and velocity spaces, a dedicated experiment has been conducted on the TCV tokamak [28] (major radius $\mathrm{R}=0.88 \mathrm{~m}$ and minor radius $\mathrm{a}=0.25 \mathrm{~m}$ ) using second harmonic X-mode $(\mathrm{X} 2) \mathrm{EC}$ waves from a $750 \mathrm{~kW}$ source. A state-of-the-art hard X-ray spectrometer [29], equipped with high-resolution digital pulse acquisition and processing [30], measures the evolution of the hard X-ray profiles with arbitrary energy and time binning. The experimental results are presented in section 2 .

In order to describe the time-evolving hard X-ray profiles, a time-varying Fokker-Planck simulation has been conducted using the 3-D bounce-averaged relativistic code LUKE [31,32], coupled with the hard X-ray synthetic diagnostic module R5-X2 [24]. A key advantage of the time-varying version of the code is that it enables modeling of non-steady-state scenarios such as modulation experiments. This in turn makes it possible to probe the plasma response while minimizing the steady-state perturbation. In the Fokker-Planck simulation, the suprathermal electron transport has been adjusted not only in the radial space but also in the momentum space, based on the time-evolving RF quasilinear diffusion coefficient. This has made it possible to assess various diffusion models by comparing the radial profile and energy spectrum of the hard X-ray measurement over time. The simulation results are presented and discussed in section 3. Conclusions can be found in section 4 .

\section{Hard X-ray measurements during ECCD discharges}

\subsection{Experimental setup}

In the present work, two ECCD discharges are analyzed: on-axis ECCD in section 2.2 and offaxis ECCD in section 2.3. All other experimental conditions were identical but the poloidal injection angle was changed slightly to move the EC wave deposition position. The EC wave trajectories and absorption locations are presented in Figure 1, and the EC wave absorption profiles are presented in Figure 2. The toroidal injection angle was in the direction such as to drive counter-ECCD to prevent an increase in plasma current. Indeed, an equivalent co-ECCD case was found to exhibit sawtooth oscillations, which compete with the imposed EC modulation and hamper its analysis.

An EC wave modulation technique was used to enhance the hard X-ray data statistics by means of conditional averaging, while keeping the perturbation to the plasma bulk properties to a minimum. During each square modulation cycle of $11 \mathrm{~ms}$, the EC wave power was ramped-up for $1 \mathrm{~ms}$ and kept at $750 \mathrm{~kW}$ for $2 \mathrm{~ms}$, followed by a ramp-down of $1 \mathrm{~ms}$ and a 7-ms low-power 

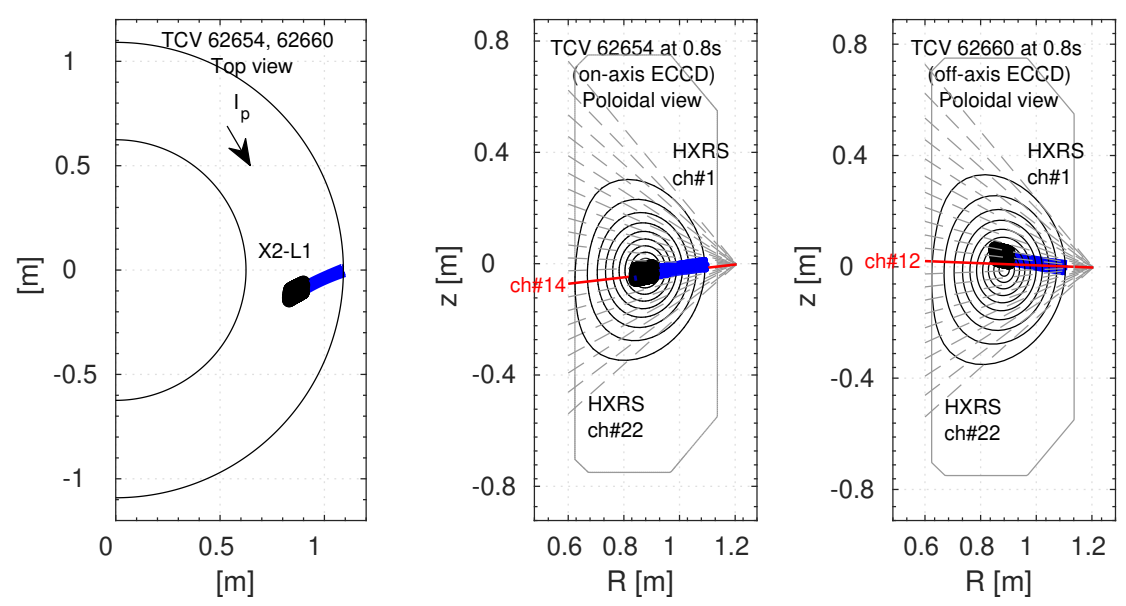

Figure 1: Top view (left) and poloidal views of the on-axis ECCD (mid) and off-axis ECCD (right) cases. EC rays are shown in blue lines and their $99.9 \%$ absorption region in thick black lines. Hard X-ray lines of sight are shown in dashed lines.

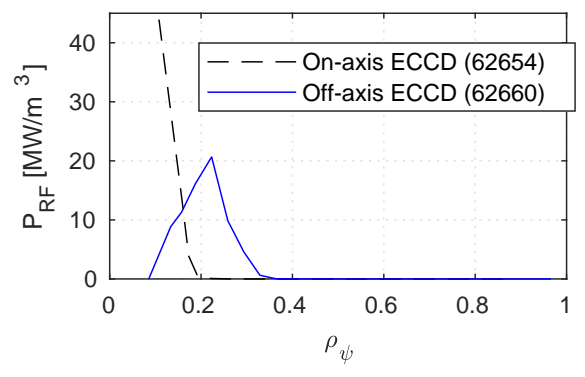

Figure 2: EC wave absorption profiles at the maximum EC wave power, calculated by the ray-tracing code $\mathrm{C} 3 \mathrm{PO}$.

phase at $100 \mathrm{~kW}$ (Figures 3 and 4). The gyrotron's characteristics preclude returning the power to zero after each high-power pulse.

The flat-top duration of $2 \mathrm{~ms}$ was determined empirically to allow sufficient photon statistics to observe the evolution of the hard X-ray profiles without perturbing the plasma significantly. If the ECCD pulse is shorter than the current diffusion time, a back electromotive force (back EMF) compensates the current change induced by ECCD [33,34], so that the perturbation on the plasma equilibrium can be neglected. This plasma reaction time can be estimated as $\tau_{\rho}=(\Delta \rho)^{2} \tau_{R}$ where the resistive time is $\tau_{R}=a^{2} \mu_{0} \sigma, \sigma$ is the electrical conductivity and $\Delta \rho$ is the current source's normalized width [33]. In this TCV discharge, $\tau_{\rho} \sim 11.4 \mathrm{~ms}$ with $\Delta \rho \sim$ 0.1. This is about 5 times longer than the pulse length of $2 \mathrm{~ms}$, thus no significant change of the current profile is expected during the ECCD period.

The ECCD modulation period of $11 \mathrm{~ms}$ was set to be long enough for the relaxation of the suprathermal electron after turning off the ECCD power, and short enough to guarantee a sufficiently large number of ECCD cycles for conclusive conditional averaging. In both on-axis and off-axis ECCD discharges, 49 pulses were used for the conditional averaging of the hard Xray data. The hard X-ray profile on the poloidal plane was measured using the equatorial hard 

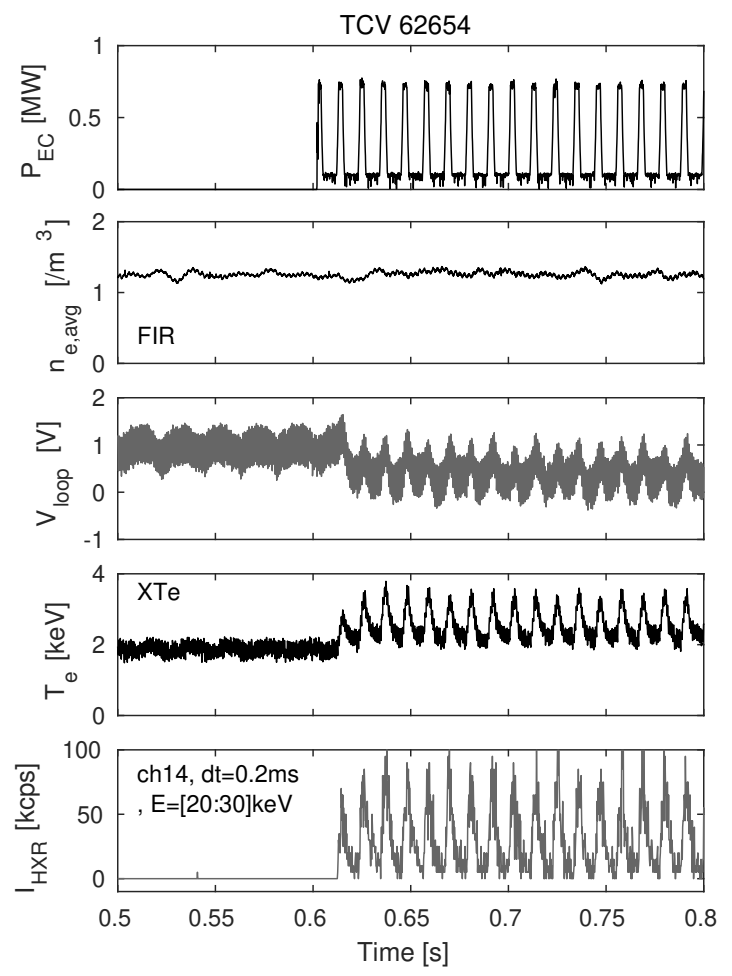

Figure 3: Time traces of EC wave power, line-averaged electron density, loop voltage, central electron temperature from soft X-ray signals, hard X-ray count rate of channel 14.

X-ray camera with 22 lines of sight (dashed lines in Figure 1). After the digital pulse processing of the collected data, the detected hard X-ray photons were sorted into time bins of $0.4 \mathrm{~ms}$ and energy bins of $10 \mathrm{keV}$.

The plasma parameters used in this work are: plasma current $\left|I_{p}\right| \sim 0.1 \mathrm{MA}$, toroidal magnetic field $\left|B_{\phi}\right| \sim 1.4 \mathrm{~T}$, central electron density $n_{e} \sim 2.0 \times 10^{19} / \mathrm{m}^{3}$ and effective charge $Z_{\text {eff }} \sim 1.2$. The electron temperature was modulated by the ECCD pulses from $\sim 2 \mathrm{keV}$ to $\sim$ $3.5 \mathrm{keV}$ in the on-axis ECCD case and from $\sim 1.5 \mathrm{keV}$ to $\sim 2.1 \mathrm{keV}$ in the off-axis ECCD case.

\section{$2.2 \quad$ On-axis ECCD}

In the on-axis ECCD case, the EC beam absorption area was localized on-axis, according to the ray-tracing calculation using $\mathrm{C} 3 \mathrm{PO}$ [31] (Figure 1). Figure 3 shows a modulation of the central electron temperature $\left(T_{e}\right)$, obtained from a soft X-ray measurement based on the absorber foil technique. The hard X-ray emission $\left(I_{H X R}\right)$ is modulated as well with ECCD. The time evolution of the line-integrated hard X-ray emission is analyzed in real and energy spaces in the next section 2.2.1, and the local emissivity is obtained using the Abel-inversion technique [35] in section 2.2.2.

No sawtooth activity was observed; however, a centrally localized frequency-chirping oscillation was found (Figure 4). The oscillation is localized near the magnetic axis and appears during the ECCD ramp-down phase. The poloidally and toroidally distributed soft X-ray array is used to analyze the mode's spatial structure, which is characterized by $m=1$ and $n=1$, where $m$ and 

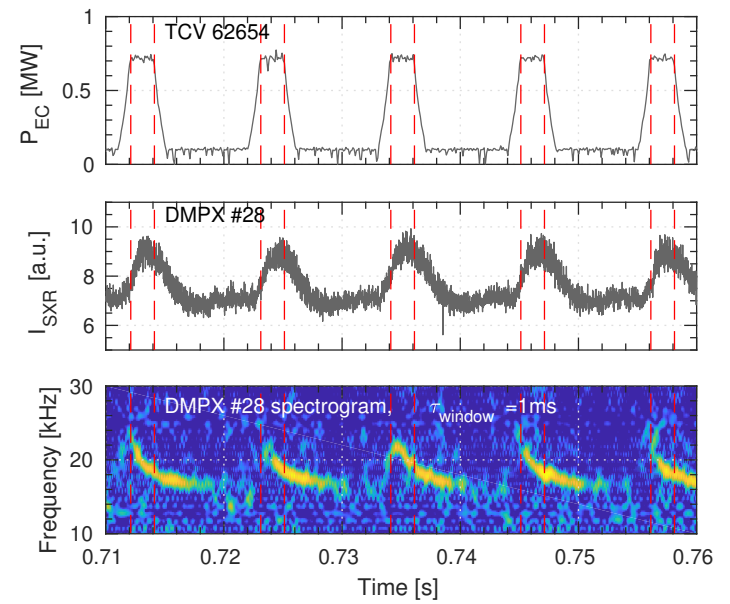

Figure 4: Time traces of EC wave power, central soft X-ray emission, and a Fourier spectrogram of the latter.

$\mathrm{n}$ are the poloidal and toroidal mode numbers, respectively. As the current profile is modified by both the ECCD and, indirectly, by the ohmic current change induced by the temperature variation (as witnessed by the loop voltage swing in Figure 3), it may be speculated that the conditions for destabilizing the internal kink mode are met transiently. This frequency-chirping mode may be an electron fishbone, which is destabilized by the energetic electrons at the $\mathrm{q}=$ 1 surface, whose location on the flux surface can vary depending on the plasma current profile change [36]. This may induce a loss of suprathermal electrons and affect the hard X-ray measurement. The nature of this mode activity and its relation to energetic electrons are left for future studies.

\subsubsection{Conditional averaging of hard X-ray data}

In order to enhance the statistics of the hard X-ray data, the count rates sorted into the time and energy bins are conditionally averaged over ECCD pulses as presented in Figure 5. For the time trace of the central electron temperature $\left(T_{e 0}\right)$, each Thomson scattering measurement point is mapped into the modulation cycle. The electron temperature increases and decreases in response to the change of the $\mathrm{EC}$ wave power.

The response of $I_{H X R}$ (hard X-ray photon count rate) to ECCD varies as a function of location and energy. Firstly the reaction of the outer channel (ch16) is slower than the central channel (ch14): for the first $1 \mathrm{~ms}$ during the ramp-up, only the central channel count rate increases significantly $(\mathrm{E}=[20: 30] \mathrm{keV})$, because $\mathrm{ECCD}$ is localized on-axis where the central channel (ch14) passes (See Figure 1). This can be also seen in the time evolution of the hard X-ray emission profiles in Figure 6. For a quantitative comparison, the ratio of the central to the outer count rate is plotted versus time in Figure 7. At the start of the ECCD pulse, the ratio increases as the $I_{H X R}$ profile forms a narrow peak. Then the ratio drops as the ECCD power increases, and the broader shape is kept until the end of the modulation cycle. This suggests outward radial transport of suprathermal electrons, which will be further studied in the following section using an Abel-inversion. 

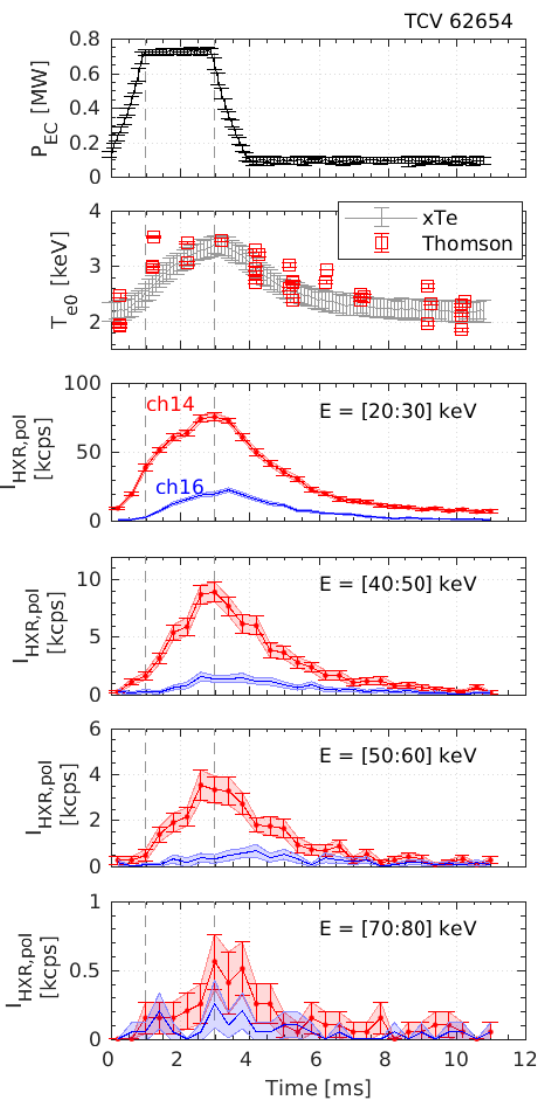

Figure 5: Time trace of EC wave power, electron temperature and hard X-ray emission $\left(I_{H X R}\right)$ on two chords from different energy bins denoted by E, averaged over EC modulation cycles.
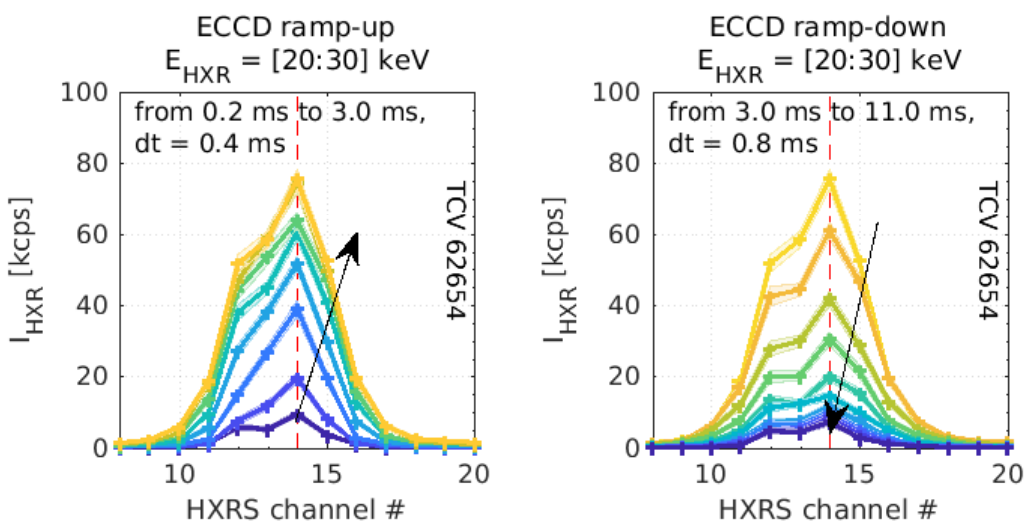

Figure 6: Time evolution of the line-integrated profile of $I_{H X R}$.

For a fixed hard X-ray channel, the $I_{H X R}$ response varies in different energy bins as well: higher energy emission responds more slowly to the ECCD power increase (Figure 5). As an example, the $\mathrm{E}=[50: 60] \mathrm{keV}$ emission increases significantly only after $1 \mathrm{~ms}$, when its $\mathrm{E}=[20: 30] \mathrm{keV}$ counterpart has already reached half of its maximum. As a working hypothesis, we may attribute this to the quasilinear diffusion of the electron distribution function in momentum space, building up in time from lower to higher energy. In order to further understand the dynamics in phase space, the experiment will be modeled using a time-dependent Fokker-Planck simulation 


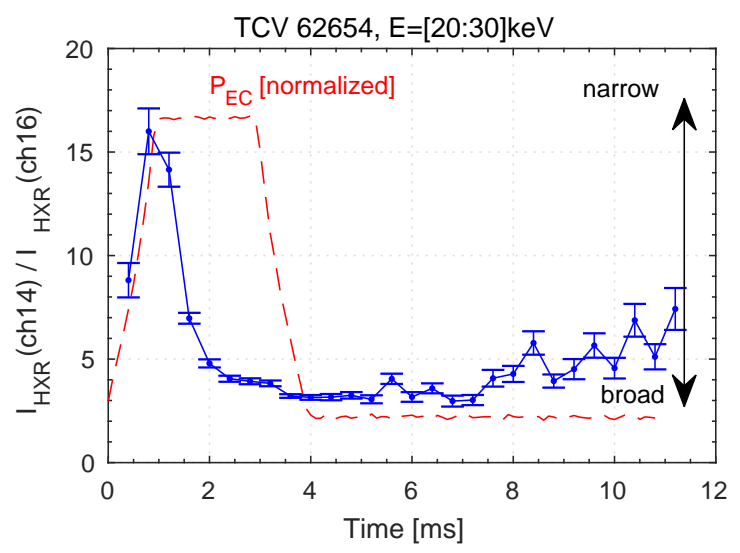

Figure 7: Blue squares: the ratio of ch14 to ch16 count rate. Red dashed line: EC wave power time trace (arbitrary units).
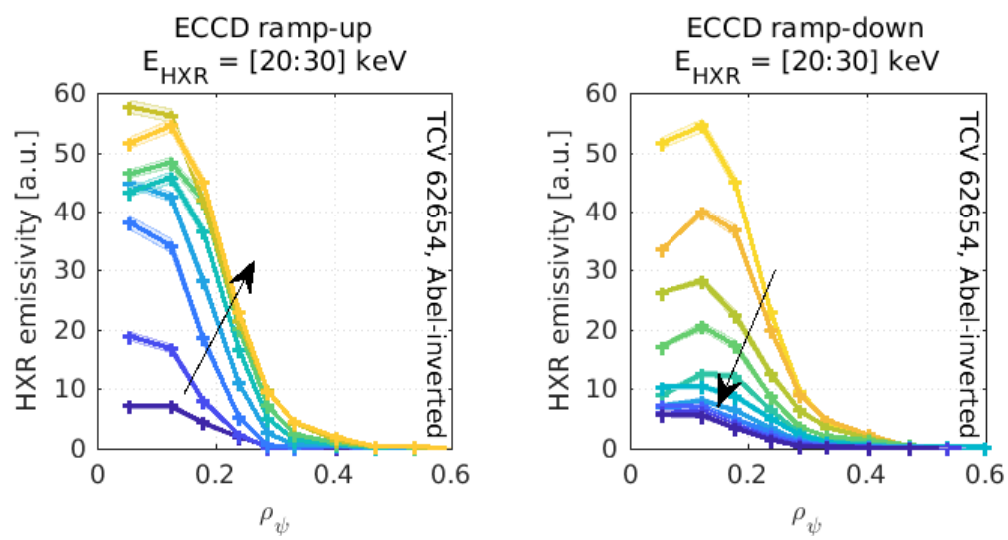

Figure 8: Time evolution of hard X-ray emissivity profile in space, Abel-inverted from the lineintegrated data in Figure 6. The time points are evenly spaced as indicated in Figure 6.

in section 3 , in combination with a hard X-ray synthetic diagnostic.

\subsubsection{Abel-inversion of hard X-ray profiles}

To derive the local hard X-ray emissivity from the line-integrated measurement, an Abelinversion method is used, assuming poloidally uniform hard X-ray emission at each flux surface. Although both the magnetic field helicity [24] and the conservation of the magnetic moment [37] can give rise to an asymmetry in the poloidal emission, this effect is negligible in tokamak plasmas with a centrally localized suprathermal electron population [24].

Figure 8 shows the Abel-inverted hard X-ray emissivity profiles in space as functions of $\rho_{\psi}$, which is the square root of the normalized poloidal flux. The temporal dynamics at different spatial locations are presented in Figure 9. The increase of the local hard X-ray emissivity is quicker in central locations (lower $\rho_{\psi}$ ) than in outer locations (higher $\rho_{\psi}$ ), as is especially clear in the normalized hard X-ray emissivity (bottom of Figure 9). This seems to indicate either an outward transport of suprathermal electrons or a growing effect of EC wave scattering. In 


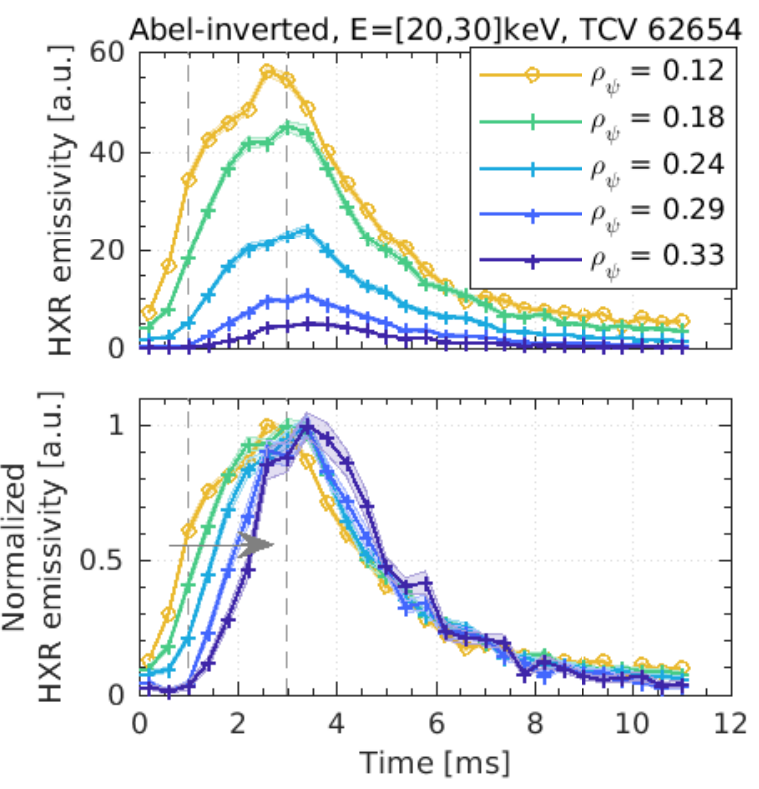

Figure 9: Time traces of local hard X-ray emissivity at various spatial locations.

the next section, the effect of diffusion will be analyzed in the Fokker-Planck modeling and a possible contribution of the EC wave scattering will be discussed.

In contrast, during the relaxation phase when only the minimum ECCD power is applied (Time $>4 \mathrm{~ms}$ in Figure 9), no significant difference in temporal dynamics has been found between the various locations. This suggests that the level of anomalous diffusion may vary with the applied EC wave power.

\subsection{Off-axis ECCD}

In order to see the effect of $\mathrm{EC}$ wave deposition location change, the poloidal angle of the EC wave was slightly moved from the on-axis ECCD discharge (Figure 1). The ray tracing calculation shows that the EC absorption is localized at $\rho_{\psi} \sim 0.2$. No MHD activity was detected in this discharge; neither the sawtooth nor the frequency-chirping internal kink mode appeared.

\subsubsection{Conditional averaging of hard X-ray data}

In the off-axis ECCD case, two peaks have been observed in the line-integrated hard X-ray emission profiles (Figure 10). The two peaks correspond to the ECCD absorption locations. While the amplitude of the overall profile changes with the applied EC wave power, the two peaks can always be distinguished. As observed in the on-axis ECCD case (Figures 6 and 7) , the response of outer channels (ch9 in Figure 11) is slower than the response of the channel whose line of sight passes the EC absorption location (ch12 in Figure 11). For the hard X-ray channels and ECCD location, see Figure 1.

Compared to the on-axis ECCD case, the overall hard X-ray count rate is lower and the energy spectrum cannot be resolved beyond $50 \mathrm{keV}$. Yet, the temporal behaviour of $I_{H X R}$ varies with energy (Figure 11), which seems to indicate an effect of RF quasilinear diffusion in the momentum space.

It should be noted that in both on-axis and off-axis ECCD cases the count rate at the lower energies does not drop down to zero at the end of the relaxation phase. This is explained by 

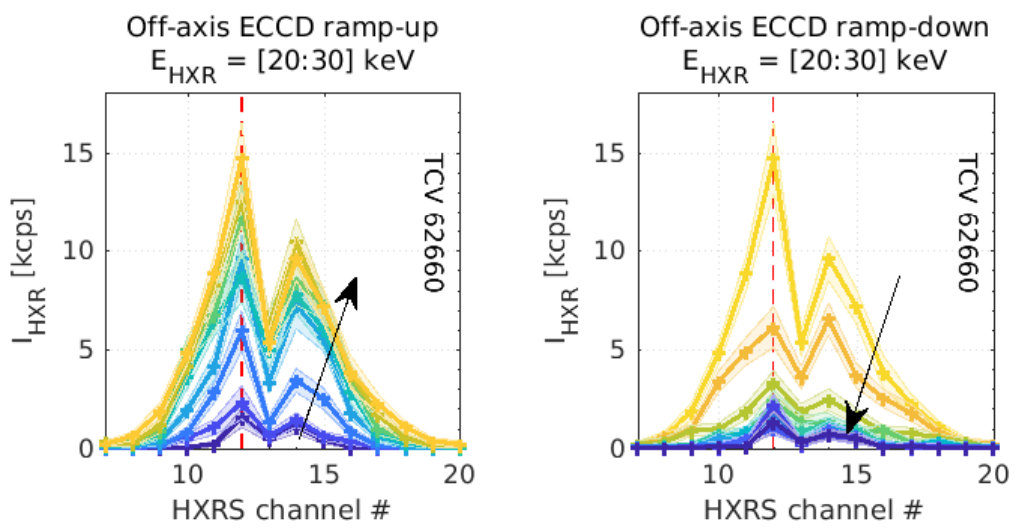

Figure 10: Time evolution of line-integrated hard X-ray emission profile with ECCD power modulation. The time points are evenly spaced as indicated in Figure 6.
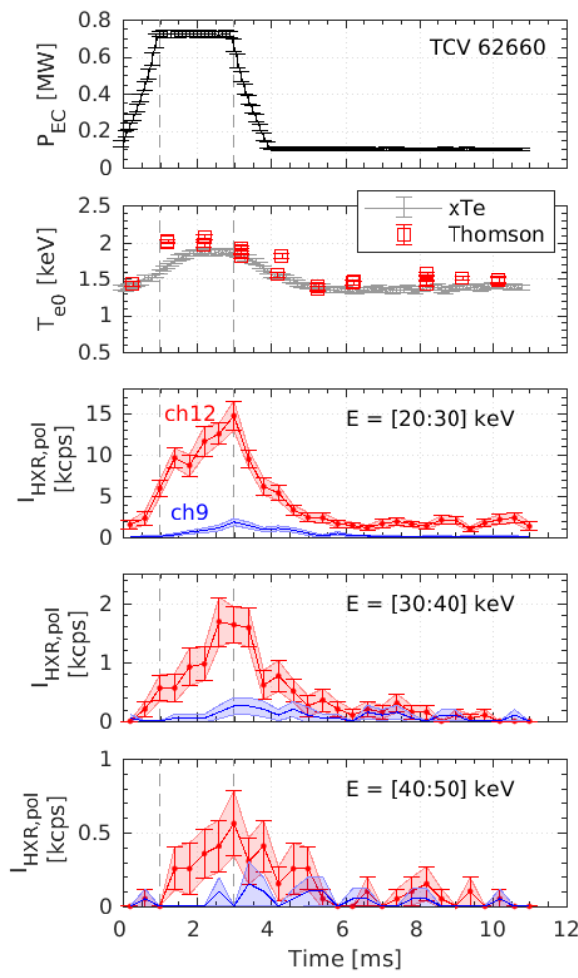

Figure 11: Time traces of EC wave power, electron temperature and hard X-ray emission $\left(I_{H X R}\right)$ in different energy bins (indicated as $\mathrm{E}$ in $\mathrm{keV}$ ), averaged over EC modulation cycles. 

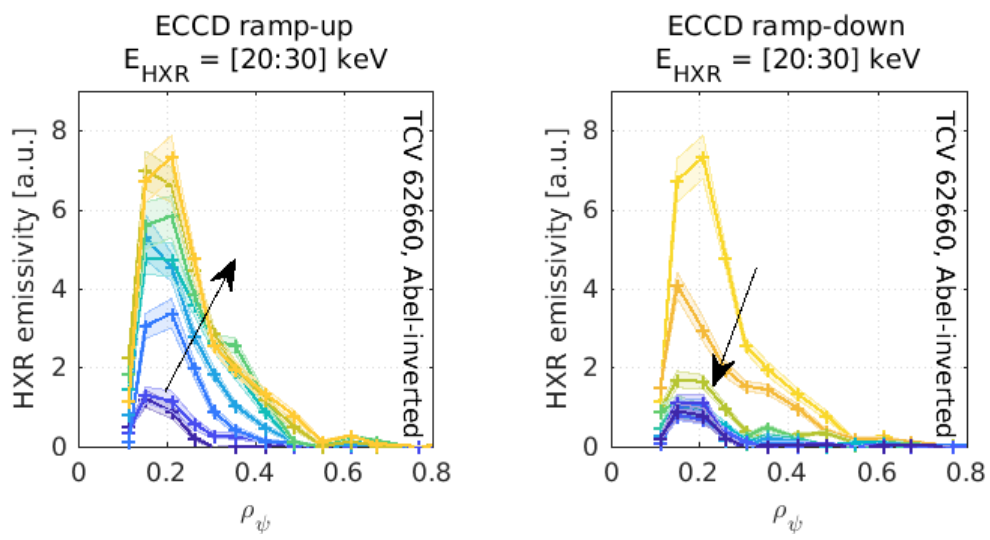

Figure 12: Time evolution of local hard X-ray emissivity profiles, Abel-inverted from the lineintegrated data in Figure 10.

the residual EC wave power held at $100 \mathrm{~kW}$ after the ramp-down. Although this power level is much lower than the maximum value $(750 \mathrm{~kW})$, the plasma is not completely ohmic and the suprathermal electron population is not negligible.

\subsubsection{Abel-inversion of hard X-ray profiles}

The Abel-inverted hard X-ray emissivity in Figure 12 shows a peak at the EC wave deposition location, $\rho_{\psi} \sim 0.2$. A broadening of the hard X-ray emissivity profile is observed, possibly due to radial diffusion or EC wave scattering, as discussed for the on-axis ECCD case. The time traces of the local hard X-ray emissivity at different locations in Figure 13 suggests that the suprathermal electrons generated at the localized ECCD location may be transported outwards over time; however, the low count rates make it difficult to observe the behavior clearly, unlike in the on-axis ECCD case.

It should be noticed that the broadening of the hard X-ray profile is not symmetric about the EC wave absorption location: the profile broadens only outwards, i.e. $\rho_{\psi}>0.2$. This suggests that the suprathermal electron transport might not be uniform in space. This will be further investigated by testing different diffusion models in the Fokker-Planck modeling.

\section{$3 \quad$ Time-dependent Fokker-Planck modeling}

\subsection{Simulation setup}

The 3-D bounce-averaged relativistic Fokker-Planck equation solver LUKE $[32,38]$ is used to calculate the time evolution of the electron distribution function $\left(f_{e}\right)$ by solving the kinetic equation including the relativistic collision operator $(C)$, the $\mathrm{RF}$ wave quasilinear operator $(Q)$, the ohmic electric field operator $(E)$, and the anomalous transport operator $(T)$ : $\partial f_{e} / \partial t=$ $C\left(f_{e}\right)+Q\left(f_{e}\right)+E\left(f_{e}\right)+T\left(f_{e}\right)$. In the anomalous transport operator, the radial transport can be controlled by modeling a diffusion parameter $D_{r}$ in $T\left(f_{e}\right)=\nabla_{r} \cdot\left(F_{r} f_{e}-D_{r} \cdot \nabla_{r} f_{e}\right)$, where $F_{r}$ describes a pinch effect. The ray-tracing code C3PO [31] is coupled to LUKE to self-consistently calculate the ray damping and the electron distribution function. The hard X-ray synthetic diagnostic module R5-X2 [24] models the bremsstrahlung emission from the distribution function calculated by LUKE and computes the photon counts at the hard X-ray 

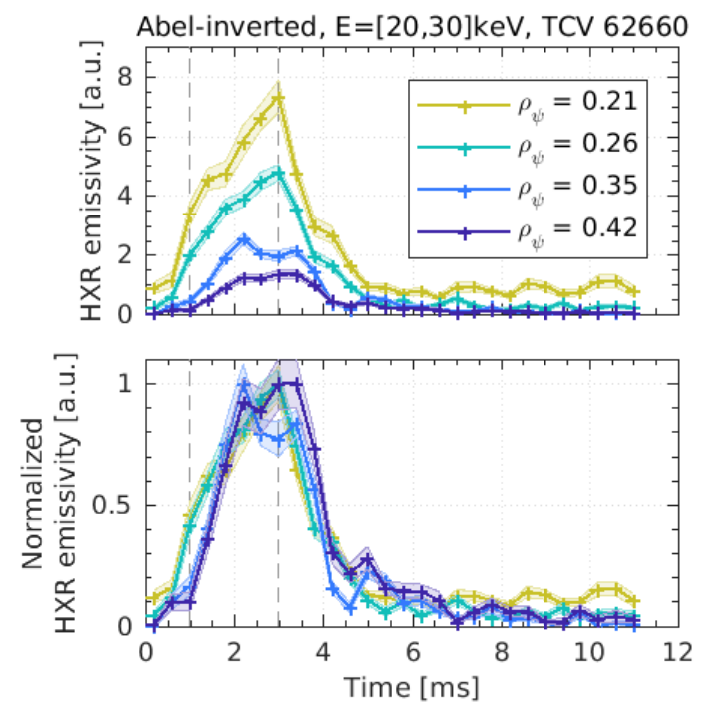

Figure 13: Time traces of the local hard X-ray emissivity at various spatial locations.

detector, allowing a direct comparison between simulation and experiment.

The electron distribution function is calculated every $0.4 \mathrm{~ms}$, which corresponds to the hard X-ray time bin length. This amounts to 28 LUKE runs per $11 \mathrm{~ms}$ experimental cycle. As observed in the experiment, the electron distribution function is not a Maxwellian at the end of the relaxed state because of the residual ECCD power. In the simulation, the electron distribution function is evolved from a Maxwellian in the first cycle, then further simulation cycles are continued until the relaxed state distribution function converges (i.e., the final state at the end of the cycle is the same for each cycle). The converged state is reached after a couple of cycles. As an example, two consecutive electron distribution functions in the momentum space are presented in Figure 14, when no radial transport of suprathermal electrons was considered.

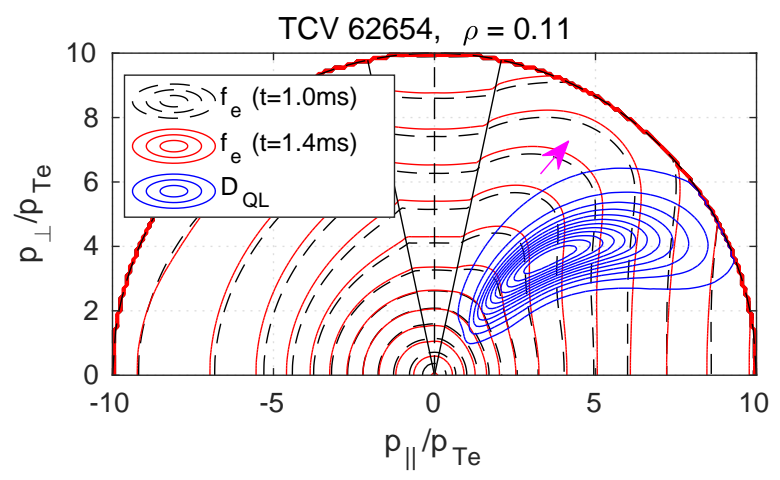

Figure 14: Electron distribution function in the momentum space at $\rho=0.11$, in the on-axis ECCD discharge, at $1.0 \mathrm{~ms}$ (black dashed line) and at $1.4 \mathrm{~ms}$ (red solid line). Blue solid line: $\mathrm{RF}$ quasilinear diffusion coefficient at $\rho=0.11$. Horizontal and vertical axes correspond to the normalized momentum, parallel and perpendicular to the magnetic field. 
Since the ECCD modulation cycle is much shorter than the resistive time, it is reasonable to assume a fixed total current profile $j_{\text {tot }}$ and adjust the back-EMF to compensate variations in ECCD. The total plasma current profile is the sum of the ohmic, RF-driven, and bootstrap current profiles $\left(j_{t o t}=j_{o h m}+j_{R F}+j_{B S}\right)$. The Fokker-Planck simulation calculates the sum of ohmic and RF-driven current profiles $\left(j_{o h m}+j_{R F}\right)$, while the bootstrap current profile $\left(j_{B S}\right)$ is calculated from the measured plasma profiles $[39,40]$. In the absence of current profile measurements in TCV, the reference current profile $j_{\text {tot }}$ is determined from time-asymptotic LUKE calculations based on the conditions at the end of the modulation cycle (magnetic equilibrium, plasma profiles, residual ECCD power), by adjusting a spatially uniform radial diffusivity to match the measured plasma current and accounting for the bootstrap current.

As presented in Figure 15, the bootstrap current profile changes during the modulation cycle following electron temperature variations. The bootstrap current value $I_{B S}$ increases from $27 \mathrm{kA}$ at $0 \mathrm{~ms}$ (beginning of the cycle) to $48 \mathrm{kA}$ at $3 \mathrm{~ms}$ (beginning of ECCD ramp-down). At each time step, the loop voltage profile is adjusted so the simulated current profile $\left(j_{o h m}+j_{R F}\right)$ matches the difference between the total and bootstrap current profiles $\left(j_{t o t}-j_{B S}\right)$. The simulation is based on the TCV magnetic equilibrium calculated by the equilibrium solver LIUQE [41].
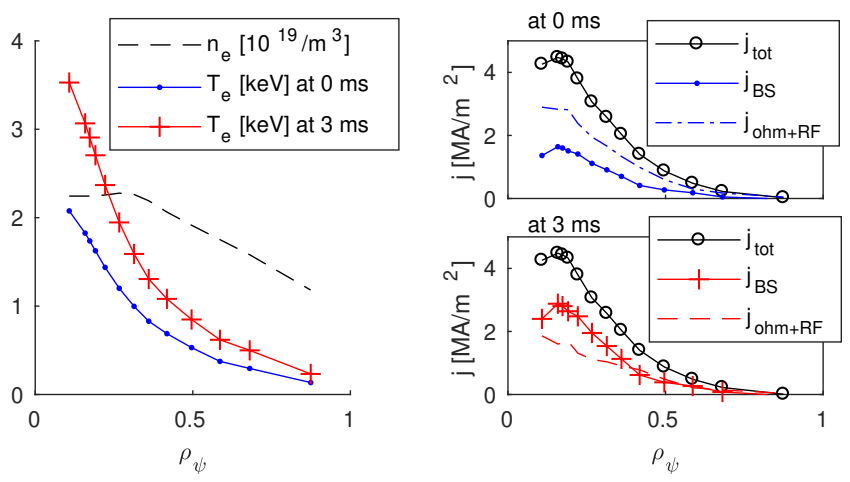

Figure 15: (Left) Electron density and temperature. (Right) Current density profiles at $0 \mathrm{~ms}$ (top) and $3 \mathrm{~ms}$ (bottom). This is the on-axis ECCD case (TCV shot 62654).

\subsection{On-axis ECCD simulation}

Figures 16 and 17 show the Fokker-Planck simulation and the synthetic diagnostic results for the on-axis ECCD discharge. As observed in previous comparisons [8,21], the modeled HXRS intensity $I_{H X R}$ is overestimated if suprathermal electron transport is neglected $(\mathrm{Dr}=0)$ in LUKE. In this section, various models of suprathermal electron transport are tested by comparing hard X-ray simulations with measurements.

Firstly, a spatially uniform diffusion model is applied to all suprathermal electrons with an energy higher than the bulk electron temperature. As illustrated in Figures 16 and 17 (Dr $=1$ ), a time-constant coefficient $D_{r}=1 \mathrm{~m}^{2} / \mathrm{s}$ yields a good match between measurements and simulations in both the hard X-ray count rate $\left(I_{H X R}\right)$ time trace (Figure 16) and energy spectrum (Figure 17) during the high-ECCD power phase (Time $<3 \mathrm{~ms}$ ). However, during the ECCD power ramp down and rest phases (Time $>3 \mathrm{~ms}$ ), the simulated hard X-ray emission is far below the measured value at all photon energies, suggesting that the suprathermal electron diffusion is much lower at that time.

If the electron transport is due to turbulence, which can be induced by the increase in the 


\section{LUKE-FEB, TCV 62654 HXRS-POL ch $14, E=[30,40] \mathrm{keV}$}

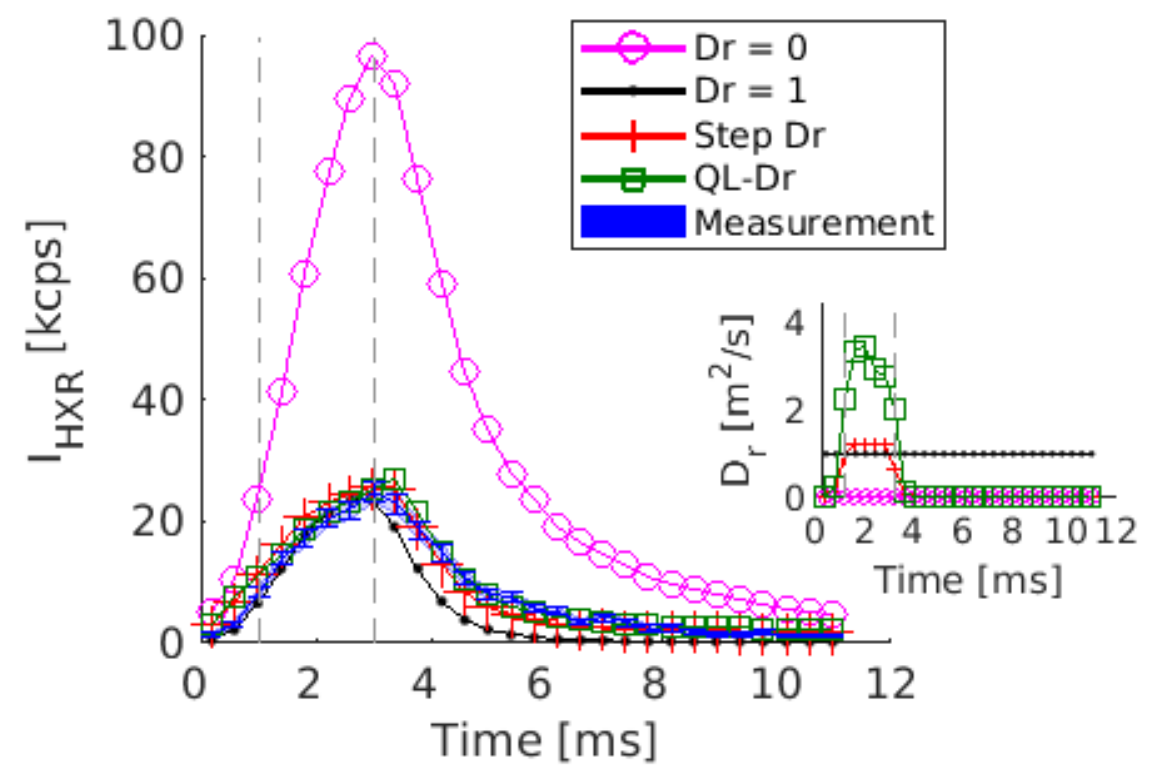

Figure 16: Simulation and experimental results for hard X-ray emission at ch14 $\left(I_{H X R}\right)$, during the on-axis ECCD discharge. Different diffusion models are also presented.
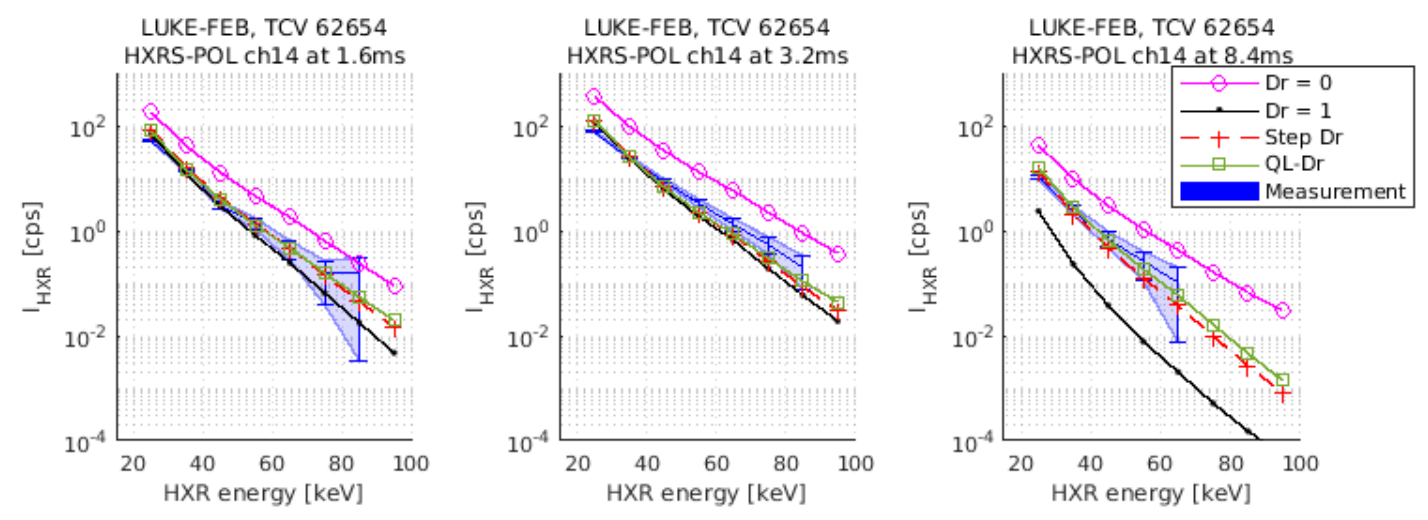

Figure 17: Simulation and experimental results for hard X-ray energy spectrum of ch14 at selected time steps, $1.6 \mathrm{~ms}, 3.2 \mathrm{~ms}$, and $8.4 \mathrm{~ms}$, during the on-axis ECCD modulation discharge.

electron temperature during ECCD, it is reasonable to assume that the transport is stronger during the ECCD phase, when the electron temperature and temperature gradients are significantly higher than during the rest phase. To test this assumption, a spacially uniform but time-dependent diffusion coefficient is constructed as a function of the ECCD power; the function chosen is a simple power dependence, with the exponent adjusted to best fit the hard X-ray measurements. Results obtained with $D_{r}(t)=1.2 \cdot\left(P_{E C}(t) / P_{E C, \text { max }}\right)^{5}$ are shown in Figures 16 and 17 ("Step Dr"). This time-step diffusion model provides a good agreement between measurements and simulations during the entire modulation cycle, which supports the hypothesis of a dependency of the suprathermal electron transport on the ECCD power level. 
Radial transport of suprathermal electrons could also result from direct EC wave-induced radial transport or from kinetic instabilities generated by the distortion of the electron distribution function from a Maxwellian under the effect of ECCD. The latter mechanism would be supported by the observation of a frequency-chirping internal kink mode as described in Figure 4. A simple way to account for either mechanism is to apply a radial diffusion coefficient that is proportional to the RF quasilinear diffusion coefficient. In this case, radial diffusion affects only resonant electrons, thereby varying in time, momentum, and configuration spaces. The resulting radial diffusion increases with the ECCD power and radial deposition profiles.

This transport model is illustrated by Figure 18 for two selected spatial locations. The RF quasilinear diffusion coefficient in the momentum space at different radial locations are scaled, to best match simulations with experimental observations. In the case of on-axis ECCD, radial diffusion (Dr in color) is only significant for the smaller $\rho_{\psi}$. Simulations using the diffusion model based on the RF quasilinear diffusion coefficient ("QL-Dr" in Figures 16 and 17) are in very good agreement with hard X-ray measurements of the time evolution and energy spectrum.
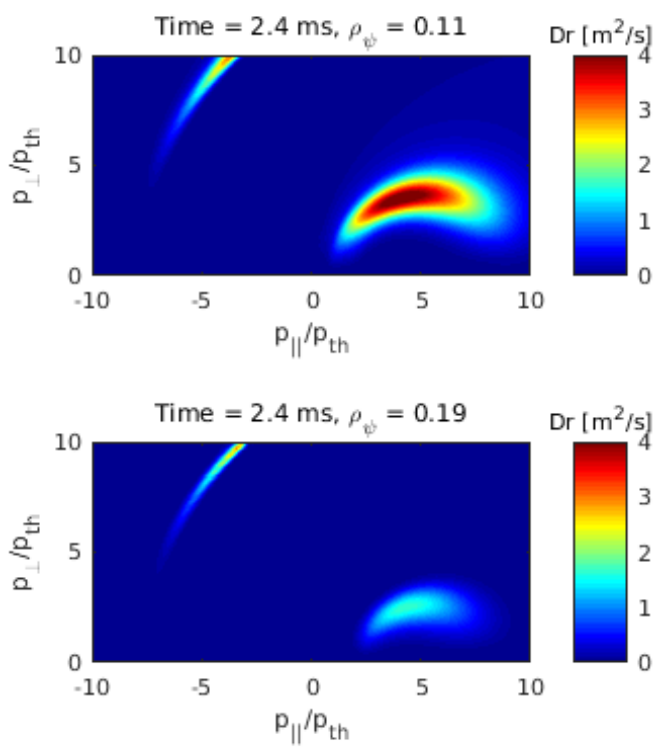

Figure 18: Radial diffusion coefficient ("QL-Dr") modeled in momentum space, in the case of the on-axis ECCD, at $\rho=0.11$ (top) and $\rho=0.19$ (bot).

Comparisons between measured and simulated hard X-ray profiles are shown in Figure 19. During the ECCD phase (Figure 19, left), the hard X-ray profile calculated with the "Step Dr" model, which applies a spatially uniform diffusion, matches the experimental profile very well. With the "QL-Dr" model, diffusion is restricted to the area of EC wave power deposition, which results in a narrower profile. During the relaxation phase (Figure 19, right), a minimum ECCD power of $100 \mathrm{~kW}$ is still applied such that a residual hard X-ray emission exists in both measurements and simulations. However, with either transport model ("Step Dr" or "QL-Dr") the diffusion is strongly reduced from the ECCD phase, which results in a simulated hard X-ray profile that is narrower than measurements.

While radial diffusion models improve the comparison between measurements and simulations significantly, it appears to be difficult to build a simple model that provides a good overall agreement in terms of hard X-ray spatial profile, time evolution, and energy spectrum. It might be necessary to resort to more complex models and also to account for the effect of EC wave 
scattering, which spreads the EC wave deposition radially.
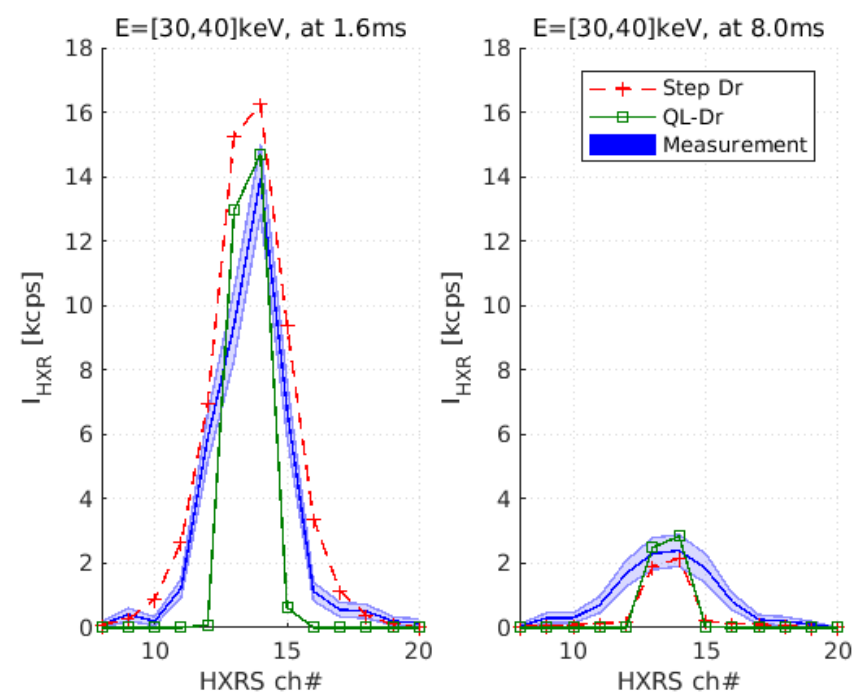

Figure 19: Hard X-ray emission profiles from measurement and simulations, in the case of the on-axis ECCD, during the ECCD phase $(1.6 \mathrm{~ms})$ and the relaxation phase $(8.0 \mathrm{~ms})$.

\subsection{Off-axis ECCD}

As illustrated in Figure 20, two distinct ECCD peaks are observed in the hard X-ray emission profile measurements of the off-axis ECCD discharge, indicating the location of EC wave power deposition. When no radial diffusion is included (case $\mathrm{Dr}=0$ in Figure 20), LUKE-FEB simulations reproduce these two peaks in the hard X-ray emission profile, but the absolute emission level is over-estimated.

The radial diffusion models described in the previous section are tested here in the off-axis case. The "Step Dr" model in which the diffusion coefficient increases with the ECCD power predicts the hard X-ray intensity, time evolution, and profile width (during the ECCD phase) relatively well. However, with a spatially uniform diffusion the two peaks are not reproduced as the profile is smoothed into a single broad peak.

The "QL-Dr" radial diffusion model is in much better agreement with the measurements as it correctly predicts the hard X-ray intensity and its time evolution. Even though the hard X-ray profile is slightly narrower than the measured one (as indicated with arrows in Figure 20), the two ECCD peaks are reproduced. Future work will include the effect of EC wave scattering to determine whether it can explain the hard X-ray emission observed in the outer channels while the two hard X-ray peaks are still resolved.

\section{Discussion and summary}

In the present work a state-of-the-art hard X-ray spectrometer in TCV has been used to analyze the time evolution of the suprathermal electron population generated by ECCD with remarkable resolution in time, space, and energy. 


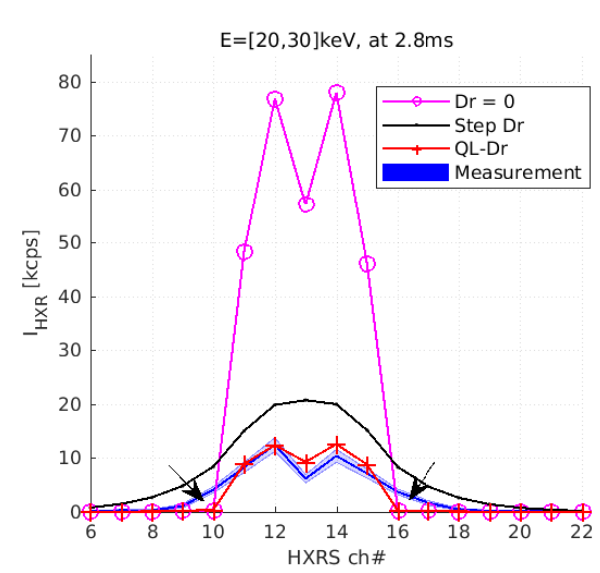

Figure 20: Hard X-ray emission profiles from measurement and simulations.

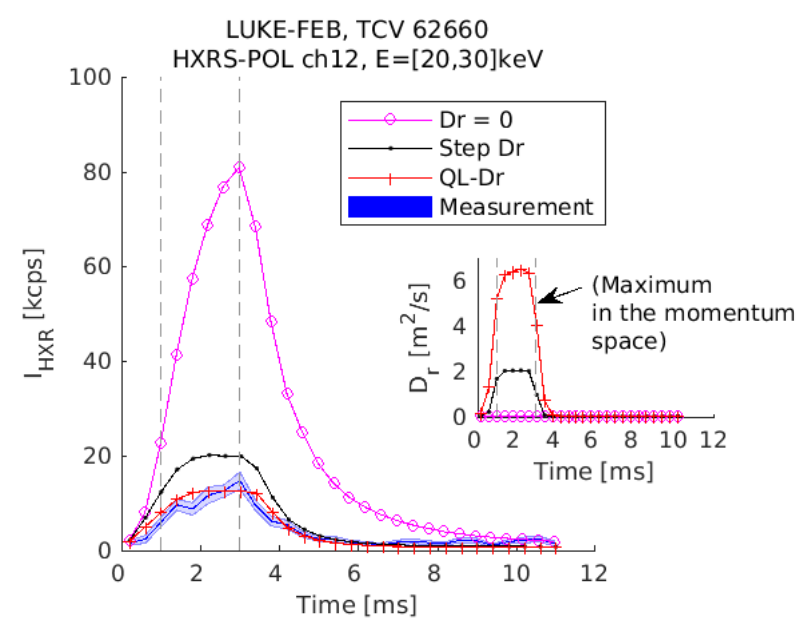

Figure 21: Simulation and experimental results of $I_{H X R}$ at ch12. Different diffusion models are also presented.

A succession of identical cycles including an ECCD phase followed by a rest phase are programmed, so that conditional averaging can be used to improve the hard X-ray measurements resolution. The cycles are long enough to allow relaxation of the suprathermal electron population generated by ECCD, but short enough to prevent any significant current diffusion, thereby simplifying the analysis.

Experimental measurements are compared with Fokker-Planck modeling coupled to a hard $\mathrm{X}$-ray synthetic diagnostic. As in previous works, it is found that the simulated emission strongly over-estimates the measurements if no radial diffusion of the suprathermal electron population is included in the calculation.

For the first time, the comparison between hard X-ray measurements and simulations is applied to a dynamic situation in which the suprathermal population builds up and then relaxes. This time evolution provides essential additional information on the suprathermal electron population dynamics, such that more refined radial diffusion models can be designed and tested by comparing with experimental observations.

It is found that the radial diffusion must depend strongly upon the ECCD power to reproduce the time evolution of the hard X-ray emission. Two models are thus defined : 1) In a first model named "Step Dr" based on the assumption that transport is due to turbulence, radial diffusion coefficient increases strongly with the ECCD power while remaining uniform in momentum and configuration spaces. 2) In a second model named "QL-Dr" suggested by the possibility that transport may be due to wave-induced transport or kinetic instabilities, the radial diffusion coefficient is proportional to the resonant interaction between EC waves and electrons, thereby varying in time, momentum and configuration spaces.

Both models can predict the hard X-ray emission, time-evolution, and energy spectrum remarkably well. However, the comparison shows discrepancies on the hard X-ray profile: the "Step Dr" model fails to show the two ECCD peaks well resolved in the measurements in the off-axis case; the "QL-Dr" model reproduces the two peaks observed experimentally, but systematically predicts a narrower hard X-ray profiles. Both models fail to reproduce the residual hard X-ray profile measured in the relaxation phase.

The effect of EC wave scattering by edge density fluctuations might also help resolve these 
discrepancies by spreading the EC wave deposition radially. Combined with this effect, the hard X-ray profiles predicted by the "QL-Dr" model might be broader. Such developments are left for future work.

\section{Acknowledgments}

This work was supported in part by the Swiss National Science Foundation. This work has been carried out within the framework of the EUROfusion Consortium and has received funding from the Euratom research and training programme 2014 - 2018 and 2019 - 2020 under grant agreement No 633053 . The views and opinions expressed herein do not necessarily reflect those of the European Commission.

\section{References}

[1] T C Luce et al. Physical Review Letters, 83:4550, 1999.

[2] S Coda et al. Plasma Physics and Controlled Fusion, 42:B311, 2000.

[3] H Zohm et al. Nuclear Fusion, 41:197, 2001.

[4] R J La Haye. Physics of Plasmas, 13:055501, 2006.

[5] TP Goodman et al. Physical review letters, 106(24):245002, 2011.

[6] T C Hender et al. Nuclear Fusion, 47:S128, 2007.

[7] R J La Haye. In EPJ Web of Conferences, volume 157, page 03027. EDP Sciences, 2017.

[8] R W Harvey et al. Physical Review Letters, 88:205001, 2002.

[9] P Nikkola et al. Nuclear fusion, 43:1343, 2003.

[10] CC Petty et al. Nuclear fusion, 42:1366, 2002.

[11] E Poli et al. Nuclear Fusion, 53:013011, 2012.

[12] N Bertelli and E Westerhof. Nuclear Fusion, 49:095018, 2009.

[13] S Coda et al. Nuclear fusion, 43:1361, 2003.

[14] N Bertelli et al. In Journal of Physics: Conference Series, volume 260, page 012002. IOP Publishing, 2010.

[15] C Tsironis et al. Physics of Plasmas, 16:112510, 2009.

[16] K Hizanidis et al. Physics of Plasmas, 17:022505, 2010.

[17] FJ Casson et al. Nuclear Fusion, 55:012002, 2015.

[18] A Köhn et al. Plasma Physics and Controlled Fusion, 60:075006, 2018.

[19] O Chellaï et al. Plasma Physics and Controlled Fusion, 61:014001, 2018.

[20] Y Peysson et al. Plasma Physics and Controlled Fusion, 53:124028, 2011.

[21] J Decker et al. In EPJ Web of Conferences, volume 32, page 01016. EDP Sciences, 2012.

[22] S Von Goeler et al. Nuclear fusion, 25:1515, 1985.

[23] G Giruzzi et al. Physical Review Letters, 74:550, 1995.

[24] Y Peysson and J Decker. Physics of Plasmas, 15:092509, 2008.

[25] S Coda. Review of Scientific Instruments, 79:10F501, 2008. 
[26] F M Poli et al. Plasma Physics and Controlled Fusion, 58:095001, 2016.

[27] A Schmidt et al. Physics of Plasmas, 18(5):056122, 2011.

[28] S Coda et al. Nuclear Fusion, 59:112023, 2019.

[29] S Coda et al. In 1st EPS conference on Plasma Diagnostics, volume 240, page 139. SISSA Medialab, 2016.

[30] J Kamleitner et al. Nuclear Instruments and Methods in Physics Research Section A: Accelerators, Spectrometers, Detectors and Associated Equipment, 736:88, 2014.

[31] Y Peysson et al. Plasma Physics and Controlled Fusion, 54:045003, 2012.

[32] J Decker and Y Peysson. Tech. rep. No EUR-CEA-FC-1736, 2004.

[33] G Giruzzi et al. Nuclear Fusion, 39:107, 1999.

[34] N J Fisch. Reviews of Modern Physics, 59:175, 1987.

[35] IH Hutchinson. Cambridge University Press, 2005.

[36] D Choi et al. Plasma Physics and Controlled Fusion, 62:025006, 2020.

[37] S Texter et al. Nuclear Fusion, 26:1279, 1986.

[38] Y Peysson and J Decker. In AIP Conference Proceedings, volume 1069, page 176. AIP, 2008.

[39] O Sauter et al. Physics of Plasmas, 6:2834, 1999.

[40] O Sauter et al. Physics of Plasmas, 9:5140, 2002.

[41] F Hofmann et al. Nuclear Fusion, 28:1871, 1988. 